\title{
JUSTICIA, TEOLOGÍA Y TEORÍA CRÍTICA EN LA OBRA TARDÍA DE MAX HORKHEIMER: REFLEXIONES SOBRE POLÍTICA RADICAL ${ }^{l}$ \\ Justice, Theology and Critical Theory in Max Horkheimer's latest work: reflections on radical politics
}

\author{
Nicolás del Valle* \\ [...]La crítica a la religión es la premisa de toda crítica \\ Karl Marx
}

\begin{abstract}
¿Remitirnos a Dios? No podemos. No podemos afirmar que exista un Dios bueno y todo poderoso. No podemos decir cómo ha venido el bien al mundo. Nosotros podemos hacer solamente una cosa: en lugar del Dogma de Dios Suscitar el anhelo de que el horror no sea la última palabra.
\end{abstract}

Max Horkheimer

Resumen

Se aborda la noción de "justicia" como eje para tematizar la relación entre "teología" y "teoría crítica" en la obra tardía de Max Horkheimer, pues, aunque no le dedicará una obra específica a este problema, se ocupará de ello en varias ocasiones transformándolo en uno de los puntos medulares a tener en cuenta cuando se pregunta por la teoría crítica, por cuanto el giro teológico de su pensamiento mostrará la radicalización de su idea de justicia y ayudará, además, a esbozar algunos lineamientos generales para una política radical.

Palabras clave: Teoría crítica, teología negativa, justicia, Horkheimer.

Abstract

These reflections treat the notion of "Justice" as a topic to stress the relationship between "theology" and "critical theory" in Max Horkheimer's latest work. Eventhough he didn't develop an specific work in regard to this issue, he empasises on it in several times turning it into one of the central points when time comes to wonder about "critical theory". In regard to the author's thesis, the theological turn of Horkheimer's thinking will show the radicalization of his idea of "Justice" and, furthermore, it will help to draw some general insights for a radical politics.

Key words: Critical theory, negative theology, justice, Horkheimer.

\footnotetext{
${ }^{1}$ Este artículo forma parte del Proyecto Fondecyt $\mathrm{N}^{\circ} 1085238$ "Nietzsche, Biopolitics and the Future of the Human", investigación sobre la relación entre hombre y naturaleza en la Escuela de Frankfurt desarrollada en el Centro de Análisis e Investigación Política (CAIP), Santiago de Chile.
} 
Nicolás del Valle

\section{INTRODUCCIÓN}

Si atendemos a la obra de Max Horkheimer luego de la vuelta a Alemania en 1949, la relación entre "teoría crítica" y "teología" es una cuestión central. Y aunque no le dedicará una obra específica a este nudo problemático, se ocupará de él en varias ocasiones transformándolo en uno de los puntos medulares a tener en cuenta a la hora de preguntarse por la teoría crítica. ${ }^{2}$ A diferencia de lo que han afirmado gran parte de los comentaristas de Horkheimer, sostengo que esta relación es patente ya desde los inicios de su carrera y no implicaría un vuelco conservador o una disonancia con su proyecto inicial. En efecto, teoría crítica y teología tendrán una relación indisoluble, pero no por ello menos problemática.

Me parece que una de las primeras entradas para tematizar la relación entre "teología" y "teoría crítica" en Max Horkheimer es la idea de "Justicia" que, en su obra tardía, será acompañada por la noción de "anhelo". Y sostengo que su idea de justicia sufre una radicalización luego de ver pasar ante sus ojos el horror de la modernidad diagnosticado en Dialéctica de la Ilustración. ${ }^{3}$ La justicia comenzará a abrirse, progresivamente, a lo que está fuera del pensamiento humano, coincidiendo con lo que llamó — junto con Theodor Adorno- "lo radicalmente Otro". El pesimismo, invocado tantas veces por él y por sus comentaristas, ${ }^{4}$ respondería a esa experiencia mundana de "horror e injusticia".

En lo que sigue, trataré de vislumbrar cómo la noción de "justicia" en el pensamiento de Horkheimer puede ser una de las entradas para abordar el problema entre teología y la propuesta teórica relacionada con la Escuela de Frankfurt, enfatizando su relevancia para una política radical. Como veremos, el "anhelo de justicia" presentado por Horkheimer será el contenido teológico de toda política que pretenda romper con la lógica inmanente de la historia. Según Horkheimer, la situación social que tienden las sociedades occidentales

${ }^{2}$ Las reflexiones sobre religión podrían encontrarse en sus primeros textos sobre metafísica de 1933 (Horkheimer, 1999); pero sólo luego de casi 30 años esta línea se pronuncia, cuando Horkheimer vuelve al problema de la teología y la influencia de Schopenhauer. Esta línea de argumentación puede ser rastreada en publicaciones aisladas, fragmentos de escritos filosóficos, aforismos, entrevistas y conferencias. Publicaciones que destacan esta trayectoria: Schopenhauer y la Sociedad (1955); La relevancia de Schopenhauer (1961); Teísmo y Ateísmo (1963); Religión y Filosofía (1967); Pesimismo hoy (1971). En habla española se publicaron algunas entrevistas, aforismos y escritos filosóficos con el título de Anhelo de Justicia. Teoría Crítica y Religión (2000). En inglés pueden encontrarse algunas de estas contribuciones en Dawn \& Decline: notes 1926-1931 and 1950-1969 (1978); Critique of Instrumental Reason: Lectures and Essays Since the End of WWII, (1974).

${ }^{3}$ La primera publicación fue de un modesto tiraje en 1944 y, luego, con algunas modificaciones considerables, fue nuevamente publicada en 1947. Trad. Española (1998).

${ }^{4}$ Sobre el pesimismo y el pensamiento de M. Horkheimer y la Escuela de Frankfurt, Cfr. Werner Post (1971), Mansilla (2008), Molinuevo (1987-88). Sobre la relación entre teoría crítica y religión, Cfr., Horkheimer, Max (2000), Mansilla (2007), Salcedo (2008), Panea (1996). 
modernas —el mundo administrado- es resultado del desarrollo histórico propulsado por el dominio de los hombres sobre la naturaleza. Su propuesta da cuenta del estatus biopolítico de la civilización, ${ }^{5}$ al presentar la dominación de la vida, la relación entre hombres y naturaleza, como el motor de la historia. Así, toda política radical frente al mundo administrado tendría un fundamento teológico y, a su vez, significaría una política de vida que pretende transformar las relaciones de dominio sobre la naturaleza.

\section{TEORÍA CRÍTICA: EL ANHELO DE JUSTICIA}

Desde su nacimiento, la "teoría crítica" se inquietaba por el avance de cierta "concepción de mundo" (Horkheimer, 2002:34), que se identificó con la teoría tradicional y, más tarde, con la racionalidad instrumental. Ya en los inicios de su trayectoria, los diagnósticos de Horkheimer sobre las sociedades modernas apuntaban a establecer una discrepancia explícita con esa concepción que funciona a favor de una sociedad de la administración capitalista. ${ }^{6}$ Las sociedades occidentales y modernas han sido marcadas por la injusticia, haciendo necesario, para toda teoría crítica de la sociedad y política revolucionaria, pensar la posibilidad de la justicia en el mundo.

En su Teoría tradicional y teoría crítica (1937), el primer texto dedicado minuciosamente a la cuestión de la teoría crítica, el otrora Director del Instituto de Investigación Social de Frankfurt establecería una estrechez entre su teoría crítica de la sociedad y la idea de justicia. ${ }^{7}$ Tal como afirma, el interés general de la teoría crítica era la "supresión de la injusticia social"; de hecho, su misma "esencia la remite al cambio histórico, a la instauración de justicia entre los hombres" (2002:77). De manera que el interés de la teoría crítica es la realización de una comunidad de hombres libres, la instauración de la justicia, mediante un cambio histórico.

Esta concepción de la sociedad se contrapone a la racionalidad instrumental que desarrolla una teoría al igual que un "sistema de símbolos puramente

\footnotetext{
${ }^{5}$ Para una lectura biopolítica de la teoría crítica de Horkheimer, $C f r$., Del Valle, (2009), Lemm (2010).

${ }^{6}$ Esta crítica de la sociedad capitalista no sólo proviene de su Teoría tradicional y teoría crítica (1937), sino que se remonta a las influencias marxistas del Instituto de Investigación que Horkheimer dirigió. Dentro de los textos que muestran este problema: The Present Situation of Social Philosophy and the Tasks of an Institut for Social Research (1931), Observaciones de Ciencia y Crisis (1932), Egoísmo y movimiento liberador (1936), Acerca del problema del pronóstico en las Ciencias Sociales (1933), Art and Mass Culture (1941), entre otros muchos. Para una buena selección de ensayos sobre el periodo temprano de su trabajo, $C f r$., Horkheimer (2003; 1993). Para la crítica del capitalismo en la teoría crítica de la Escuela de Frankfurt, Honneth (2009).

${ }^{7}$ Sobre esta relación entre teoría crítica y justicia en otros filósofos de la escuela de Frankfurt, Cfr., Panea (2001).
} 
matemático" y como "resultado de la abstracción", sostiene Horkheimer (2002:2325). Al contrario de esta "teoría tradicional de la sociedad", que comprende a la realidad social dividida en átomos o partes, la racionalidad crítica construye una teoría orientada a la emancipación de los hombres y a la "transformación de la totalidad", agrega (2002:43), pensando a la sociedad integralmente como un todo. Toda aproximación a la justicia, desde la concepción instrumental, pasa por una reducción al mero cálculo matemático o económico, denotando que la idea de justicia defendida por la teoría crítica se opone a una noción de justicia distributiva o económica. De hecho, uno de los grandes problemas de la teoría tradicional, donde el positivismo es el emblema, es que, debido a sus pretensiones de neutralidad, difícilmente puede proveer una reflexión moral o de justicia al analizar las problemáticas sociales (Horkheimer, 1989:105; Adorno y Horkheimer, 1969:13).

Sin embargo, luego de su Teoría tradicional y teoría crítica —donde la posibilidad de la instauración de la justicia entre los hombres era patente- la visión de Horkheimer se desliza hacia una crítica aún más radical, ya no sólo del capitalismo, sino de la civilización humana en su totalidad. El diagnóstico de la sociedad moderna, luego del ascenso del fascismo, demuestra que la justicia se hace imposible, pero necesaria desde un punto de vista crítico, en el mundo terrenal. En efecto, mientras que en el primer momento de la teoría crítica existía un optimismo explícito respecto de los logros de la teoría crítica y de la política radical, luego de la Dialéctica de la Ilustración (1944-47) las posibilidades de abolir la injusticia son cada vez menores.

En este momento de su obra, el mundo de los hombres, lo existente, se halla regido por la injusticia y la dominación. En este escenario, si bien Horkheimer se pregunta por la realidad existente, una teoría de la sociedad con pretensiones críticas no trabaja al servicio de ella, como lo haría la versión tradicional, sino que tiene como meta pronunciar su secreto (2002:51). Ciertamente, el horror y la injusticia reinan en el mundo de los hombres, pero la teoría crítica se inclina por pensar que ambas no tienen la última palabra. El teórico crítico tendrá como horizonte pensar lo Otro que ha sido mitigado a lo largo de la historia de la civilización. Esta actitud es la que, para Horkheimer, coincide con la religión: el horror y la injusticia que atraviesan este mundo no tienen, necesariamente, la última palabra. La diferencia, en todo caso, estriba en que la religión presenta a la justicia como mandato, mientras que la teoría crítica como un anhelo de que no quede todo definitivamente en la injusticia (2000:217).

\section{TEOLOGÍA NEGATIVA}

Luego de las múltiples declaraciones de Horkheimer sobre la religión, las críticas a sus propuestas no tardaron en aparecer. Las acusaciones sobre su preocupación por la religión oscilarán desde tildarla como la "resignación del típico intelectual revolucionario" hasta un "retroceso conservador" (Estrada, 
(1990, 1989, 1987; Molinuevo, 1987-88), ambas contradictorias con su obra temprana. Contrariamente a esto, intentaré esbozar las líneas de fuerza que coinciden con su primer proyecto, distinguiendo la noción horkheimeriana de la teología, pues, como veremos, la religión que reivindica se dirige al núcleo detrás de toda religión, pero que, luego de la hegemonía del cristianismo y la modernidad, ha tendido a desaparecer.

Ciertamente, las nociones de teología y religión que reiteradamente utiliza en su pensamiento tardío no se corresponden con gran parte de las opiniones de la tradición revolucionaria. Horkheimer reconoce fuertemente el rol de dominación social que precedió al arribo de la modernidad y que ha permanecido en las religiones en la historia de las sociedades occidentales; sin embargo, no será el único sentido que verá luego de su deconstrucción de la teología. De hecho, como él mismo distinguiría en uno de sus aforismos titulados ¿Qué es Religión? (2000) hay que diferenciar los sentidos de la religión; uno auténtico y otro no auténtico, una religión en buen sentido y otra en mal sentido. En el fondo, el malentendido que hay tras la resignación y conservadurismo que se achaca al giro ético-teológico de Horkheimer radica en la distinción de los sentidos que cohabitan en su noción de religión.

Horkheimer intenta revelar el secreto que se encuentra al interior de la religión, pero cautivo desde el avance del cristianismo. Siguiendo la tradición revolucionaria, hace ver en varias oportunidades lo falso de las religiones, asintiendo el gran problema de toda teología positiva. El cristianismo, por ejemplo, dice mucho sobre Dios, lo presenta "como dogma positivo" y como "objeto de saber y de posesión" (2000:136) al describirlo (potencialmente, al menos) por un conjunto de características o al afirmar tajantemente su existencia (ya sea por convicción o por medio de hechos o evidencias constatables). Esta "religión no-auténtica" afirma la "vana mentira de que el mal, el sufrimiento, el horror tienen un sentido", enfatiza (2000:226) y, en ella, la función social de Dios es actuar como un "mero disgregador" (2000:136).

Por otro lado, el sentido "auténtico" de la religión que rescata es la que plantea "el inextinguible impulso, sostenido contra la realidad, de que ésta debe cambiar, que se rompa la maldición y se abra paso a la justicia" (2000:226). Esta es la noción que debería contener toda teoría crítica y política radical. Contrario a las posturas más tradicionalistas, según Horkheimer, hay que salir de aquella visión de la religión que la endosa necesariamente con el conservadurismo y a la imagen de la autoridad, pues, según su manera de ver no se tiene en cuenta lo poderosamente revolucionaria que puede ser la referencia a "Dios" - aunque siempre haya preferido referirse a lo "radicalmente otro"- luego de un análisis crítico. He aquí el punto de contacto entre la teoría crítica y su noción de teología: la 
contribución al debate teológico político que hace Horkheimer, como él mismo lo acepta, es la referencia a ese "anhelo de justicia"

El Anhelo de que la realidad del mundo con todo su horror no es lo último que une y relaciona a todos los hombres que no pueden o no quieren resignarse a la injusticia de este mundo (2000:136).

He aquí cómo su concepto de teología es acompañado por su noción de "anhelo" en contraposición al "dogma", pues, mientras éste ultimo se pronuncia y asiente sobre la existencia de lo absoluto (ya sea negando o afirmando la existencia de Dios, como ocurre con el ateísmo o el teísmo respectivamente), la teología de Horkheimer tiene la esperanza de ello, pero nunca la seguridad y la certeza. En consecuencia, contrario a varios de sus comentaristas, al dar cuenta del giro teológico de la filosofía Horkheimer no se caería en una regresión conservadora sino, más bien, revelaría que ya en la teología encontramos una posibilidad radical. La teología, tal como la define, es el anhelo de que "la injusticia que atraviesa este mundo no sea lo último, que no tenga la última palabra" (2000:106).

En este periodo de su pensamiento, en Horkheimer la idea de justicia ya tiene un matiz teológico, aunque sólo al ser acompañado por su noción de anhelo, termina por concretar su idea negativa de la teología. A diferencia del "dogma", el "anhelo" no describe positivamente ese estadio absoluto de justicia. Siguiendo las huellas de la fenomenología, Horkheimer sostiene que los humanos son seres finitos y sólo tienen acceso a esa finitud, haciendo imposible cualquier conocimiento del "Absoluto"; como máximo, los seres humanos podrían acceder a lo que "Dios" no es. De ahí la apelación a la teología negativa. La influencia del judaísmo en el pensamiento del teórico se hace evidente, pues Dios, al igual que la justicia, es irrepresentable en el mundo de los hombres. ${ }^{8}$ Horkheimer recuerda que la Biblia dice: "no debes hacerte ninguna imagen de Dios" (2000:195).

Los hombres son finitos y el mundo en el cual se desenvuelven es una representación histórica socialmente condicionada, es decir, relativa; mientras que, al contrario, la imagen de Dios coincide con un "radical afuera" imposible de representar y que Horkheimer identifica con lo infinito y absoluto. De ahí que Adorno y Horkheimer hayan decidido hablar de lo "totalmente Otro" (2000:195). Esta táctica permitirá concebir a "Dios" como una alteridad radical, fuera de la razón representacional y el lenguaje

\footnotetext{
${ }^{8}$ Las menciones a los judíos y al judaísmo son innumerables a lo largo de su obra y, sobre todo, luego del fin de la Segunda Guerra Mundial. Para una relación entre Justicia, lo Otro y los judíos, Cfr., Diner (1993:335-363).
} 
humano. ${ }^{9}$ De hecho, si es que existe algo así como Dios es una alteridad que no puede ser comprendida o absorbida por la racionalidad del sujeto.

Contrario a lo que Jürgen Habermas ha planteado (2001:122-134), Horkheimer no cae en una postura metafísica con su giro teológico. Al contrario, su pliegue hacia el léxico de la teología y metafísica occidental es motivado por su pretensión crítica de declararla contradictoria y, por ello, imposible para la vida humana y su mundo. Horkheimer no afirma la existencia de una potencia trascendente, más allá del mundo. ${ }^{10}$ Más bien, intentará demostrar que caer tanto en su afirmación como en su negación es apresarse en el dogma. No se pronuncia sobre la existencia de Dios pero, sí, sobre nuestra experiencia como seres humanos en el mundo.

Toda la argumentación de Horkheimer se dirige a extirpar el dogma y la imagen positiva de Dios y ubicar al anhelo en el centro de la religión. El motivo es claro, tal como lo aprecia Adorno en su correspondencia con Horkheimer: una de sus inquietudes centrales es pensar la justicia y su realización en un mundo tan injusto como el de los hombres. ${ }^{11}$ Uno de los resultados de la Ilustración, de aquel weberiano "desencantamiento del mundo" (Weber, 2003:167-188; 234-236), es que con la extinción de la religión a manos de la razón, se declara la carta de defunción del pensamiento de la justicia.

EXCURSO SOBRE POLÍTICA, AMOR Y TEOLOGÍA EN EL ANHELO DE JUSTICIA DE MAX HORKHEIMER

En el contexto de la discusión sobre la obra de Horkheimer, la acción moral consiste en hacer algo bueno por el otro con la esperanza de que aquella acción haga más bella nuestra existencia. La acción moral es, ante todo, para y por el otro, demostrando que el cuidado de éste ya implica el cuidado de sí mismo. Esto no se debe a que una instancia superior o trascendente recompensará la buena acción, sino que deriva de si el otro encuentra alegría

\footnotetext{
${ }^{9}$ En dicho estadio de justicia consumada se encontraría la "verdad última" que "no puede traducirse al lenguaje y mundo conceptual humano", Horkheimer (2000:135-136).

${ }^{10}$ En un discurso con motivo del centenario de la muerte de Arthur Schopenhauer, Horkheimer sostendrá que la actualidad de su doctrina radica en que, sin caer en la resignación, "priva al mundo del engañoso fundamento dorado que le confería la vieja metafísica" (56, la cursiva es mía) y "no se deja nublar la mirada por nacionalismos fanáticos o por teorías de una justicia trascendente" (57). Incluso afirma que "ninguna calamidad será compensada en un más allá", pues, si es que existiera algo así como una compensación de la calamidad, correspondería a los hombres (2000:56-57). Ciertamente el fundador de la teoría crítica no desecha nociones como lo Eterno, Infinito, Absoluto o Trascendente, pero, según nuestra lectura, no lo hace, pues se propone, a través de su uso, mostrarlas imposibles para la realidad humana. Sobre la obra tardía de Horkheimer y esta postura postmetafísica, Cfr. Sánchez (2001:223-246).

11 "Lo primero en ti fue la rebelión contra la injusticia". T. Adorno, Carta de 14 de febrero de 1965 en Horkheimer (1996:594. Mi traducción).
} 
en dicha acción. En consecuencia no es el pensamiento de Dios lo que cualifica una acción como moral, sino que es "sencillamente el hecho de que mi vida, aun cuando deba sacrificarse por el otro, es embellecida por las reacciones de éste" (2000:113). El lema diría algo así como "Si el otro es feliz, también yo lo soy".

La idea de Justicia que se encuentra en el último Horkheimer se radicaliza a tal punto que se enlaza con la imagen misma de la comunidad impolítica, como es el amor. De manera que toda política que presente a la Justicia como su horizonte, hunde sus raíces en el anhelo del otro, en el anhelo de la persona amada (2000:113). El problema es que las sociedades modernas transitan hacia un mundo administrado que no conoce el amor, negando al otro y a la Justicia. Horkheimer esgrime, siguiendo a Schopenhauer, que en el mundo de los hombres, donde impera el horror y la injusticia, reina el pecado original; vale decir, la afirmación del propio yo y la negación de los otros (2000:109).

Entonces ¿Cómo salvar su idea de justicia entre los hombres? Como vemos, las sociedades no conocen la justicia, puesto que se niega al otro y sólo se busca la autoconservación y afirmación del yo. La vía entregada por Horkheimer estriba en la posibilidad de una práctica teórica y política que apele a lo diferente y sostenga el anhelo de que la transformación de nuestras sociedades hacia un "mundo Otro" es posible. Su gesto apunta, también, a rescatar cierto rasgo teológico pues, sin ello, toda política sería absorbida por la racionalidad instrumental del cálculo y la administración. En palabras del propio Horkheimer: "Una política que, aunque sea de forma refleja, no contenga en sí la teología, se reduce --por hábil que sea- en último término, a negocio" (2000:105).

Esto será muy bien desarrollado en el conjunto de conferencias dictadas por Horkheimer en 1944 que fueron publicadas bajo el título de Eclipse of Reason o Crítica de la razón instrumental. ${ }^{12}$ En su fase tardía, el avance de aquella formalización de la razón resultará en que nuestro mundo administrado "puede apropiarse incluso de la crítica, del arte negativo, de la resistencia y usarlos como ornamentos" (2005:60), reduciendo toda política a simple racionalidad instrumental. Es el contenido teológico, la apelación a lo imposible, "anhelar la justicia", lo necesario para toda política que pretenda transformar la totalidad de las relaciones humanas y consumar la justicia entre los hombres. Si bien no es posible afirmar o negar la existencia de lo imposible (pues se caería en el dogma), lo cierto es que la justicia se muestra como necesaria e imprescindible para toda política emancipatoria.

\footnotetext{
12 La primera edición del libro fue en 1946, pero trabajamos aquí con la segunda edición alemana de 1967. Trad. española (1973).
} 
En rigor, aquella política de la que hablamos se hace cargo del núcleo de la religión: el anhelo de la justicia absoluta, infinita y universal, pero teniendo siempre claro lo imposible de su realización. Imposible, pero necesaria. La cuestión que plantea Horkheimer sobre la justicia es crucial, pues, aun reconociendo que el lugar de la justicia es el de lo imposible, ésta debe erigirse como una exigencia tanto para vivir con los otros como para el mismo teórico crítico. Su noción de justicia se juega siempre entre los hombres y hacia el otro. Un anhelo de justicia que, traducido teológicamente, se ha comprendido como el "Amor al prójimo"13

La conciencia de la injusticia procede (...) en último término de la teología, de la religión; pues allí se dice: "Ama al Prójimo como a ti mismo", allí se establece la justicia como una exigencia (2000:217).

Consecuentemente, cualquier persona que no cree en Dios, pero trata al otro con amor y justicia, es también religioso, lo cual demuestra que el rol de la religión es necesario si se vive en comunidad. Sólo de este principio de "amar al prójimo" puede desprenderse una moral humana y una noción de justicia, lo cual señala que si queremos vivir con otros este componente religioso debe estar presente. El problema de las sociedades contemporáneas es que en ellas domina una racionalidad que cosifica al otro o que existen formas teológico-metafísicas positivas que rinden culto dogmáticamente a un Dios trascendente, negando, ambas, toda posibilidad de justicia. "El amor y la amistad - argumenta Horkheimer- descansan en la idea de la propia finitud" pero, a su vez, se orienta a lo absoluto (2000:204). La política que podría desprenderse de acá es siempre desde la finitud, pero abriéndose hacia el afuera del mundo humano.

\section{EL MUNDO ADMINISTRADO Y LA DOMINACIÓN DE LA NATURALEZA}

El giro teológico en el que se encuentra el pesimismo que ha caracterizado al pensamiento tardío de Max Horkheimer responde — como lo notará Foucault más tarde- a las reflexiones sobre su presente. ${ }^{14}$ La impresión que tenía de la situación social de su tiempo sería la de un tránsito, la de una "sociedad en transición" (1986) cuyo motor es el progreso de la racionalización formal a partir de la dominación de la naturaleza. Al pensar su presente, Horkheimer lo caracteriza como "fase de transición hacia la sociedad absolutamente

\footnotetext{
13 "Es posible que la traducción "Ama a tu prójimo como a ti mismo" no sea correcta, que más bien debería decir (...): "Ama a tu prójimo; él es como tú”, Horkheimer (2000:108).

${ }^{14}$ Me refiero al gesto hacia Hegel, Nietzsche, Weber y la escuela de Frankfurt cuando discute la pregunta kantiana ¿Qué es la Ilustración?: "Una ontología de la actualidad (del presente) es una forma de filosofía que desde Hegel a la escuela de Frankfurt, pasando por Nietzsche y Max Weber, ha fundado una forma de reflexión en la que intento trabajar", Foucault (1991:207).
} 
racionalizada" (2000:205), lo que llamó el "mundo administrado". Como bien lo supo, su presente, al estar entrecruzado por el pasado, procede ya antes que el surgimiento del capitalismo.

Este juicio se ubica en la así llamada "ruptura" con el proyecto original de la teoría crítica de comienzos de la década del 30' y que se reconoce en la Dialéctica de la Ilustración (1998) escrita en conjunto con Theodor W. Adorno. Aquí, desde sus inicios, el problema central de la civilización humana ha sido el hombre como dominador de la naturaleza, el núcleo donde aparece el problema político de la vida: el desarrollo de la humanidad ha sido un gran proceso civilizatorio que ha negado la vida de la naturaleza para proteger y conservar la vida humana.

Tal argumento se encuentra en los orígenes de la civilización con la historia de Odiseo develando el miedo a la naturaleza, miedo a lo otro, como piedra angular de dominio y punto constitutivo de la subjetividad occidental: En la base del mito de la Ilustración se aloja el miedo a la "naturaleza", a la dominación que ésta podría ejercer sobre el hombre (1998:97-128). Por tal razón, la emancipación del hombre — según el iluminismo - sólo se logra con la dominación de la naturaleza. El hombre de ciencia, en tanto dominador de la naturaleza, se relaciona con las cosas en la medida que las manipula y administra, siendo éstas un simple sustrato de dominio.

La dialéctica es consumada con la dominación del hombre por el hombre. La dominación sobre la naturaleza (ya sea la "naturaleza externa" del mundo circundante, como la "naturaleza interna" al sujeto) resulta en la propia auto-dominación de los seres humanos, erigiendo un mundo injusto que administra la vida humana y natural. ${ }^{15}$ Esta dominación sobre la vida de la naturaleza empuja a las sociedades hacia un estadio de vigilancia $y$ administración ${ }^{16}$ que garantice la autoconservación de la especie humana.

Al interpretar de esta manera la obra de Horkheimer, la dominación de la naturaleza es constitutiva a la injusticia del mundo administrado. La dominación de la naturaleza, y la injusticia, tiene una dimensión global que abarca desde el medio ambiente hasta la sociedad en su conjunto, mercantilizando la vida de la naturaleza y de los hombres al ser absorbida por la racionalidad instrumental.

En definitiva, con la llegada de la modernización toda sociedad concluiría en una sociedad de la administración donde la especie humana se enseñoree,

\footnotetext{
15 Un ejemplo: "Con la píldora, la industria farmacéutica moderna ha convertido a la fuerza reproductiva humana en una fuerza manipulable; un día necesitaremos también una administración de nacimientos", Horkheimer (2000:115).

16 "Con la ciencia y la técnica ha sometido el hombre bajo sí las inmensas fuerzas de la naturaleza. Si queremos que estas fuerzas no actúen de manera destructiva, deben ser puestas bajo la vigilancia de una administración central verdaderamente racional”, Horkheimer (2000:205).
} 
pero, también, donde el individuo singular no signifique nada, donde el individuo sigue "convencionalmente todas las órdenes y toda las reglas" (2000:216-217), demostrando el ocaso de libertad del individuo y la afirmación de la masa pasiva y regularizada. En estas sociedades donde el mercado reina, la relación entre los hombres y de ellos con la naturaleza es reducida al negocio y a la conveniencia, sentenciando a la moral y la política a su muerte.

El pesimismo se hace patente. Luego del horror vivido en todo el siglo $\mathrm{XX}$, al filósofo se le hace imposible pensar a la Justicia entre los hombres. En nuestro mundo reina una forma de pensar y de actuar que niega al otro y la vida de la naturaleza y justifica un conjunto de tecnologías que tienen como objetivo el control, la vigilancia y la administración de la vida. Frente a esto, lo único que nos queda es anhelar lo Otro, esa idea de justicia y verdad última que se aloja fuera de lo humano. Lo poderoso de esta cuestión es que la justicia no se corresponde con el mundo humano sino que, al final de su pensamiento, Horkheimer la ubica fuera de lo posible.

Una de las salidas que propongo a este juicio crítico de Horkheimer es una política que busque transformar las sociedades modernas mediante una práctica teórico-política que anhele la realización de la justicia y luche contra la dominación de la naturaleza. Una de las posibles políticas --a partir de este punto- consistiría en la afirmación de la vida en su totalidad mediante la problematización de la frontera entre hombre y naturaleza. Mientras que la racionalidad instrumental del sujeto establece un corte en la continuidad de la vida entre hombre y naturaleza, para lograr su auto-constitución, la racionalidad crítica y sustantiva excedería toda subjetividad abriéndola hacia una "alteridad radical".

La dominación sobre la vida de la naturaleza podría ser limitada con la liberación del pensamiento crítico y el recuerdo de ello; es decir, una política de la naturaleza que fecunde la vida y se abra al otro. En contraste con esa racionalidad instrumental que avanza junto a la mercantilización de la vida, la política de la que habla Horkheimer, se construye sobre una racionalidad sustantiva que piensa la totalidad de la vida que excede a la especie humana.

La política de la naturaleza que se podría desprender de la propuesta teológica de Horkheimer consiste en una lucha contra la "realidad dada", lucha que pretende su transformación hacia un estadio de Justicia y conciliación - siempre imposibles - entre hombre y naturaleza pero, al mismo tiempo, un duelo por la injusticia que cruza la historia de las sociedades. ${ }^{17}$ Con todo, un atisbo de una política radical que anhele una sociedad mejor, es decir, que haya superado la injusticia.

\footnotetext{
17 "Aun cuando una sociedad mejor haya superado la injusticia presente, la miseria pasada no será reparada ni superado el sufrimiento de la naturaleza circundante”, Horkheimer (2000:111).
} 
Nicolás del Valle

\author{
Universidad Diego Portales* \\ Instituto de Humanidades \\ Res.: Ejército 278, Piso 1 (patio interior), Santiago (Chile) \\ D. P. 8370056 \\ nicolasdelvalle.o@gmail.com
}

\title{
BIBLIOGRAFÍA
}

Adorno, Theodor; Horkheimer, Max. Dialektik der Aufklärung. Frankfurt, AM: Fischer, 2001.

------ Dialéctica de la Ilustración. Madrid Trotta, 1998.

------ La Sociedad. Lecciones de Sociología. Buenos Aires: Proteo, 1969.

Benhabib, Seyla; Bonss, Wolfgang; John Mccole. (Eds.). On Max Horkheimer: New Perspectives. Cambridge, M. A.: MIT Press, 1993.

Del Valle, Nicolás. "Biopolítica, ecología y razón instrumental: consideraciones en torno a Max Horkheimer y Michel Foucault", en Revista Pléyade, Centro de Análisis e Investigación Política, $N^{\circ}$ 3, Chile (2009):1-24.

Estrada, J. A. La teoría crítica de Max Horkheimer; del socialismo ético a la resignación. Granada: Universidad de Granada, 1990.

"La prevalencia de Schopenhauer sobre Marx en la Teoría Crítica de Horkheimer", en Revista Pensamiento, Vol. 45, № 177 (1989).

------ "La "última" Teoría Crítica de Max Horkheimer", en Revista Pensamiento, Vol. 43, No 171 (1987).

Foucault, Michel. Saber y Verdad. Buenos Aires: La Piqueta, 1991.

Habermas, Jürgen. Israel o Atenas. Ensayos sobre religión, teología y racionalidad. Madrid: Trotta, 2001.

Honneth, Axel. Patologías de la razón. Historia y actualidad de la teoría crítica. Buenos Aires: Katz, 2009.

Horkheimer, Max. Eclipse of Reason. New York: Oxford University Press, 1947.

----- Crítica de la Razón Instrumental. Buenos Aires: SUR, 1973.

------ Critique of Instrumental Reason: Lectures and Essays Since the End of WWII. New York: Seabury Press, 1974.

------ Dawn \& Decline: notes 1926-1931 and 1950-1969. New York: Seabury Press, 1978.

------ Sociedad en transición: estudios de filosofía social. Barcelona: Planeta Agostini, 1986.

------ Between Philosophy and Social Science: Selected Early Writings. Cambridge, MA: MIT Press, 1993.

------ Gesammelte Schriften, XVIII. Frankfurt, 1996.

------ Materialismo, metafísica y moral. Madrid: Tecnos, 1999.

------ Anhelo de Justicia. Teoría Crítica y Religión. Madrid: Trotta, 2000.

------ Teoría tradicional y teoría crítica. Barcelona: Paidós, 2002.

------ Teoría Crítica. Buenos Aires: Amorrortu, 2003. 
Sociedad, razón y libertad. Madrid: Trotta, 2005.

Lemm, Vanessa. "Critical Theory and affirmative biopolitics: Nietzsche and Domination of nature in Adorno/Horkheimer", en Journal of Power, $\mathrm{N}^{\circ} 1$, Vol. 3, april (2010):75-95.

Mansilla, H. C. F. "Reflexiones sobre el sentido de la historia: entre el optimismo doctrinario y el pesimismo", en Revista de Filosofía, Vol. 26, № 59 (agosto 2008) 23-41.

------ "Religión y razón como factores de complementación y colaboración", en Revista de Filosofía, Vol. 25, № 55 (2007):87-102.

Marcuse, Herbert; Popper, Karl; Horkheimer, Max. A la búsqueda del sentido. Salamanca: Sígueme, 1989.

Marx, Karl. Crítica de la Filosofía del Derecho de Hegel. Santiago: Clinamen, 2009.

Molinuevo, José Luis. "El tardío pesimismo metafísico de Horkheimer", en Revista de Filosofía, Vol. 1, № 3 (1987-88):115-126.

Panea, José Manuel. Querer la Utopía: Razón y autoconservación en la Escuela de Frankfurt. Sevilla: Universidad de Sevilla, 1996.

------- "Filosofía como búsqueda de la Justicia (A propósito de M. Horkheimer, T. W. Adorno y H. Marcuse)", en Contrastes Revista interdisciplinar de filosofía, Vol. VI (2001):99-112.

Post, Werner. Kritische Theorie und metaphysischer Pessimismus. Zum Spätwerk Max Horkheimer. Munich, 1971.

Salcedo, José Arturo. "Horkheimer: tres momentos de la crítica a la modernidad", en Revista Intersticios, Vol. 2, No 2 (2008):15-23.

Sánchez, Juan José. "Compasión, política y memoria. El sentimiento moral en Max Horkheimer", en Revista Isegoría, № 25 (2001):223-246.

Schmidt, Alfred; Altwicker, Norbert (Ed.). Max Horkheimer heute: Werk und Wirkung. Frankfurt: Fischer Taschenbuch, 1986.

Stirk, Peter. Max Horkheimer: A new interpretation. Boston: Barnes and Noble Books, 1992.

Weber, Max. La ética protestante y el espíritu del capitalismo. México: F. C. E., 2003.

Wiggershaus, Rolf. Max Horkheimer zur Einführung. Hamburg: Junius, 1998. 